\title{
Industrial Automation Information Analogy for Smart Grid Security
}

\author{
Muhammad Asif ${ }^{1}$, Ishfaq Ali ${ }^{1}$, Shahbaz Ahmad ${ }^{1}$, Azeem Irshad ${ }^{2}$, Akber Abid Gardezi ${ }^{3}$, \\ Fawaz Alassery ${ }^{4}$, Habib Hamam ${ }^{5}$ and Muhammad Shafiq ${ }^{6, *}$
}

\author{
${ }^{1}$ Department of Computer Science, National Textile University, Faisalabad, 37610, Punjab, Pakistan \\ ${ }^{2}$ Department of Computer Science and Software Engineering, International Islamic University, Islamabad, Pakistan \\ ${ }^{3}$ Department of Computer Science, COMSATS University Islamabad, Islamabad, Pakistan \\ ${ }^{4}$ Department of Computer Engineering, College of Computers and Information Technology, Taif University, Taif, \\ Saudi Arabia \\ ${ }^{5}$ Faculty of Engineering, Moncton University, NB, E1A3E9, Canada \\ ${ }^{6}$ Department of Information and Communication Engineering, Yeungnam University, Gyeongsan, 38541, Korea \\ *Corresponding Author: Muhammad Shafiq. Email: shafiq@ynu.ac.kr \\ Received: 25 August 2021; Accepted: 27 September 2021
}

\begin{abstract}
Industrial automation or assembly automation is a strictly monitored environment, in which changes occur at a good speed. There are many types of entities in the focusing environment, and the data generated by these devices is huge. In addition, because the robustness is achieved by sensing redundant data, the data becomes larger. The data generating device, whether it is a sensing device or a physical device, streams the data to a higher-level deception device for calculation, so that it can be driven and configured according to the updated conditions. With the emergence of the Industry 4.0 concept that includes a variety of automation technologies, various data is generated through numerous devices. Therefore, the data generated for industrial automation requires unique Information Architecture (IA). IA should be able to satisfy hard real-time constraints to spontaneously change the environment and the instantaneous configuration of all participants. To understand its applicability, we used an example smart grid analogy. The smart grid system needs an IA to fulfill the communication requirements to report the hard real-time changes in the power immediately following the system. In addition, in a smart grid system, it needs to report changes on either side of the system, i.e., consumers and suppliers configure and reconfigure the system according to the changes. In this article, we propose an analogy of a physical phenomenon. A point charge is used as a data generating device, the streamline of electric flux is used as a data flow, and the charge distribution on a closed surface is used as a configuration. Finally, the intensity changes are used in the physical process, e.g., the smart grid. This analogy is explained by metaphors, and the structural mapping framework is used for its theoretical proof. The proposed analogy provides a theoretical basis for the development of such information architectures that can represent data flows, definition changes (deterministic and non-deterministic), events, and instantaneous configuration definitions of entities in the system. The proposed analogy provides
\end{abstract}

This work is licensed under a Creative Commons Attribution 4.0 International License, which permits unrestricted use, distribution, and reproduction in any medium, provided the original work is properly cited. 
a mechanism to perform calculations during communication, using a simple concept on the closed surface to integrate two-layer cyber-physical systems (computation, communication, and physical process). The proposed analogy is a good candidate for implementation in smart grid security.

Keywords: Industry 4.0; information architecture; industrial automation

\section{Introduction}

Information and Communication Technology (ICT) is penetrating all aspects of industrial and manufacturing systems, accelerating the generation of large amounts of industrial data. Moreover, Industry 4.0 (the fourth industrial revolution) reduces the human factor, relying entirely on data to show the importance of industrial data. We need to make better use of data in industrial manufacturing systems [1]. The underlying data needs to be transformed into valuable information. Similarly, intelligent services need to be integrated with manufacturing systems to support the decision-making process. In addition, it is expected that future manufacturing systems will be sufficiently efficient, flexible, competitive, sustainable, safe and reliable [2]. They must be designed to reduce waste of materials, capital, energy, and media. The ability to improve control, reconfiguration, optimization, and human-computer interaction in the manufacturing process is also crucial for future manufacturing [3]. The challenge of managing data can turn it into useful knowledge and help us make smart and reliable decisions [4].

Industry 4.0 is actually an extension of the cyber-physical system, realizing decentralized intelligence in the manufacturing field through the end-to-end value chain of the Industrial Internet of Things (IIoT) [5]. The Industry 4.0 manufacturing system has the most advanced planning and innovation capabilities in the fields of product design, smart medical, remote control, service and diagnosis, condition monitoring, prototyping and development, active and predictive maintenance, tracking and tracing, agility, real-time applications, etc. [6]. All technologies in Industry 4.0 need to be viewed from a perspective where integration is crucial. A networked industrial environment or intelligent manufacturing unit is the realization of the Industry 4.0 revolution. Recently, Industry has changed many manufacturing concepts, and it is expected that there will be major changes in the next few years. Bitkom stated that after the introduction of Industry 4.0, by 2025, a total of 267 billion euros may increase the total revenue of Germany [7], which is the next breakthrough in the business. These advances have an impact on information exchange and management. The complexity stems from the fusion of at least 30 different technologies.

Industry 4.0 essentially requires strict connections between various devices and the data flow between these devices to make decisions. Data generating devices are usually sensors, RFID tags, Internet Protocol (IP)-based cameras and many other types of cameras that generate large amounts of data. In addition, in order to achieve robustness, multiple devices are used to record the same phenomenon, that is, a large number of devices are deployed to obtain basically the same information [8]. Therefore, a computing layer is needed to calculate this initial data and transmit the required information. In this regard, we need to eliminate redundant data and reduce a considerable amount of data. To perform this calculation, it takes a small amount of time, which increases the transmission delay, which is the potential for real-time automated production and many other [9]. Future industrial systems need to be designed to reduce the waste of resources such as energy, processing time, materials, 
capital, manpower, and media. In this regard, modern computing technologies such as the IIoT, big data, autonomous robots, and human-computer interaction can play an important role in improving control, automatic reconfiguration, and optimization in the manufacturing process, and empower future manufacturing [10].

For industrial automation, different sensing and driving mechanisms are involved in a centralized environment (a single machine or an entire factory). These sensing and automation mechanisms generate data consistently like the flow of water from the source to the reservoir. The key to the success of industrial automation depends on the speed of determining and responding to changes. Data generation equipment plays a key role in identifying changes along with the effectiveness of the agent function's response. An effective industrial automation system, including many other features, must be able to effectively sense changes and act accordingly. To meet the needs of industrial automation, event-driven architecture (EDA), service-oriented architecture (SOA) and list information architecture (IA) have been developed. All these solutions are based on the best practices of software engineering, namely the three layers of change, communication, calculation and execution. However, the pace of change in real-world industrial automation, especially the nature of Industry 4.0 standards, requires intensive configuration, reconfiguration, and real-time response to changes. Therefore, this can be regarded as the most difficult real-time level of change detection, reconfiguration and driving method.

The modern industrial environment can be visualized as a situation, and data is generated spontaneously and non-spontaneously in huge quantities with hard real-time constraints. Data generation resources are inherently diverse, so data. The visualization of industrial data in the context of Industry 4.0 reflects special requirements, namely (a) the environment is constantly changing or evolving. Therefore, data generation takes the form of a stream; (b) Instant configuration of all devices in the process; (c) In order to process robust data, some kind of calculation is required during communication. This analogy maps the natural phenomenon of electric charges placed in a closed surface, where sensing/monitoring nodes are placed in the environment. This analogy provides a theoretical basis for the development of industrial environmental information architecture. This research helps to establish a theoretical model of information flow in a smart device grid based on the electrical flux through any system. This model provides a mechanism for the instantaneous information flow, spontaneous configuration and reconfiguration of smart grid nodes. It also provides a new way to build and organize information specific to the environment and the smart grid of industrial plants. This research helps provide instant and dynamically configurable solutions by spontaneously exchanging information for state-of-the-art Industry 4.0 systems.

For ease of understanding, we use an example of a smart grid, where the information requirements are difficult to implement in real time. Smart grid system is a conceptual power system that can realize bidirectional current. Its digital communication supports real-time detection, reaction, proactive action on any changes in the entire system, and self-repair capabilities. It can also communicate instantly with suppliers and consumers. This research paper provides an analogy in which a point charge causes current to pass through a charged hollow sphere. Each node is similar to a point charge, and its surface is similar to the next layer of nodes. Electric flux is similar to the communication channel between nodes. This analogy is based on the fact that every Gaussian sphere retains the same charge as Barry.

This analogy provides a theoretical basis for the development of IA based on the physical phenomenon of "the electric flux passing through a hollow sphere (Gaussian sphere) due to a point charge". Because the IA of the smart grid system needs to meet multiple requirements of information, so that the system can detect, react and actively respond to changes in the system at the same speed 
when power occurs. This analogy will provide the basis for the development of this type of information architecture, which has the ability to report electrical phenomena at the rate of occurrence. Although designing information architecture for a specific requirement requires an in-depth analysis of the requirements, the system provides a more general idea for building the IA of this type of system.

The rest of this article is organized as follows. Section 2 summarizes the background. Section 3 discusses the system model. Section 4 introduces the mapping of the proposed analogy. Finally, the discussion and conclusion are presented in Sections 5 and 6, respectively.

\section{Background}

With the emergence of Industry 4.0, industrial automation has become a mixture of multiple technologies and concerns [11]. In the paper [12], we can find different aspects that can be considered for industrial automation (for example, cloud computing, cognitive computing, network security, Internet of Things (IoT), big data, etc.), as shown in Fig. 1. With the increase in product categories and the shortening of product life cycles, the manufacturing process is currently undergoing a paradigm shift. The impact of this phenomenon on them is that they must manufacture highly personalized products with short delivery times and low production costs [13]. In this case, it is necessary to adjust the production steps used to assemble or process the product according to the product requirements. In addition, the movement of materials between machines and assembly stations requires a more flexible logistics framework. Material flow systems (MFS) that cover different transference paths related to the required product design evolution, configuration, and reconfiguration can be very complex and require constant adjustments [14]. Despite user customization, assembly automation itself is a highly variable environment due to multiple factors that occur at the highest frequency. In order to cope with faster data generation speed and spontaneous changes, professional information architecture is necessary.

We give a brief introduction to the information architecture in the following.

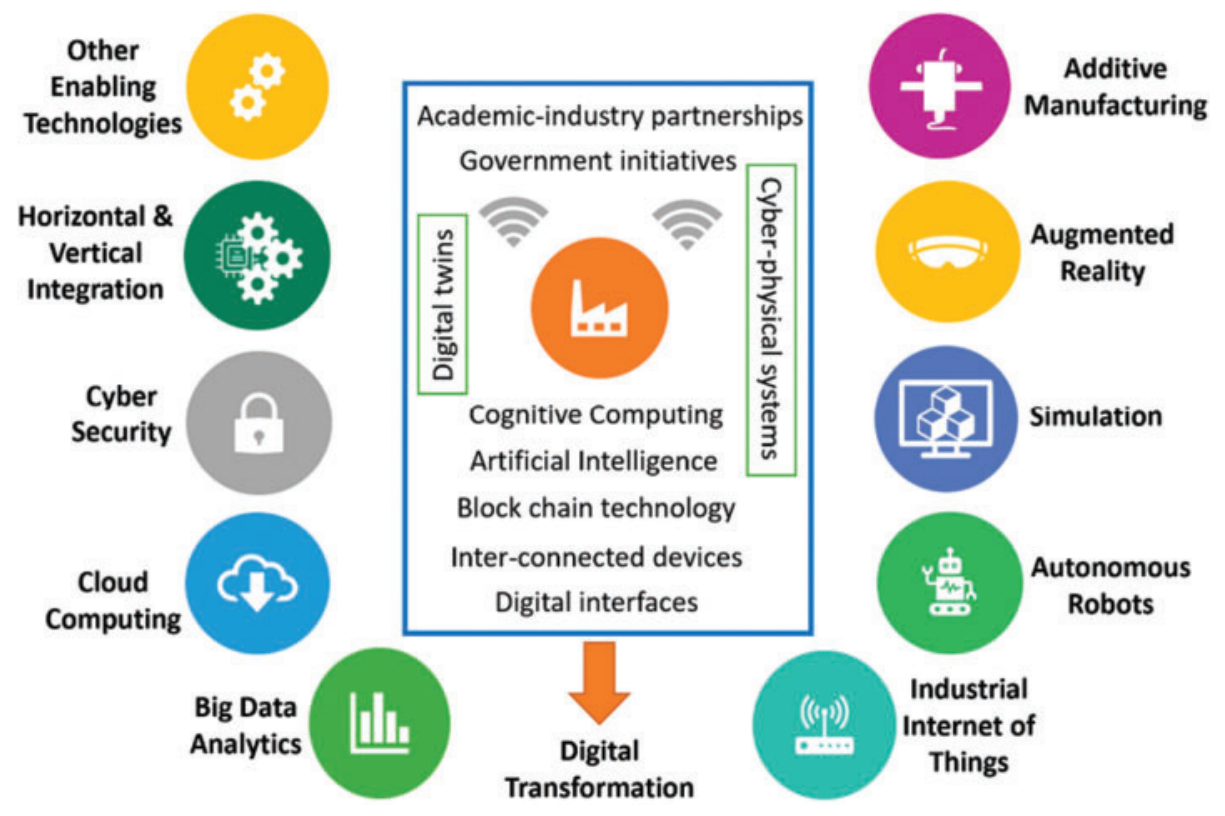

Figure 1: Industry 4.0 technology pillars [12] 


\subsection{Information Architecture Model}

The information architecture model defines the rules for information access, presentation, maintenance, and interconnection. The key concepts related to it include "packages." Packages are called basic atomic information pieces, which can be stored, presented, and accessed efficiently and accurately. Attributes are additional details of an entity, whether physical or conceptual [15]. We can find various information and communication architectures in the fields of manufacturing research and automated factories, which describe some common design goals [11,12]. This includes data conversion to achieve efficiency in material loss, capital investment, energy demand, and media utilization to achieve efficient, flexible, safe and sustainable production. Some other goals include improved control, automatic reconfiguration, optimization, and human-computer interaction involved in the manufacturing process. Below are brief details of some important information architecture point-to-point $(\mathrm{P} 2 \mathrm{P})$, service-oriented architecture (SOA), event-driven architecture (EDA), and line information system architecture (LISA).

\subsubsection{Point-to-Point Architecture}

Traditionally, manufacturers tend to use a client-server architecture that is, adding every new hardware/function to the server. Each node of the client has information about the server, and the server has information about the client. The client passes the information to the server, and the server relaxes the prescribed policy to the required client. This architecture considers a legacy system, which hardly meets current requirements. The high cost of adopting modern systems forces companies to use this information model.

\subsubsection{Service-Oriented Information Architecture ( $S O A$ )}

Applications with self-control act as "services", and each service interacts with each other and operates based on the latest information received. The key features that make SOA popular are loose coupling and greater flexibility. Due to the improvement of infrastructure and the optimization of business processes, it has attracted the attention of academia and industry. Although the genre of SOA has completely changed the business process, it is combined with the business process, so it pays more attention to strict timing and resource requirements. The utility of SOA in real-time projects, where changes or events are considered as a function of time, as the prediction of changes is limited; it takes longer to start a specific service.

\subsubsection{Event-Driven Architecture (EDA)}

The centralized control of SOA means obtaining services by restricting loose coupling through the request control mechanism. Although the later version of SOA2 provided publishing events to obtain services, which reduced the centralization of services, a centralized mechanism was still needed to control services and execute them through events. Therefore, an event-driven architecture has been proposed, centering on events rather than services. Events are generated from perception, without considering how and where they will be processed. EDA is more loosely coupled and distributed in control than SOA.

\subsubsection{Line Information Structure Architecture (LISA)}

LISA is essentially an EDA integrated through messages. The core component of LISA is the "message bus", which provides a format and delivers messages through service endpoints. This allows events to be loosely converted into information. Prototype technology is used to create messages 
from events. This architecture specifies a prototype for each type of event, and then spread it further through the message bus. LISA provides loosely coupled and distributed systems and its integration between services through the message bus (the limitation of EDA), but considers deploying a cyberphysical system to monitor an environment. The system treats sequences as events, and spends a small amount of time in sensing and computing, and further communication through messages, that is, sensing, computing and communication have been completed especially when it is done simultaneously in a strict real-time environment, because reality changes within a fraction of a second. There are milliseconds between the occurrence of an event and the communication and configuration. For the implementation of Industry 4.0, cyber-physical systems are the key technology; a brief description is given in the following.

\subsection{System Model}

Here we discuss some incompetent terms, such as nodes, surfaces, events, agents, and important functions to grasp the concepts in this research. The node is an atomic sensing mechanism $\mathrm{N}$ deployed for the focused environment $\mathrm{E}$, capable of sensing a single quantity $\mathrm{Q}$ with a series of values. For example, deploy a node to sense temperature and have a sensing capability from 0 to 180 degrees. For an analogy, it is defined as the point charge enclosed in the surface, which is responsible for the charge distribution on the entire surface. The surface is a receiver, responsible for spontaneously receiving sensory data, and for interpreting the instantaneous state of environment $\mathrm{E}$ from the sensory data of node $\mathrm{N}$. The configuration of the entire system is affected by the state of node N. Since the node is deployed in a spontaneously changing environment, its instantaneous state has an impact on the reconstruction of the entire system. Therefore, the receiver is defined as a surface with a charge distribution on its surface, and any changes in the point charge or around the charge will affect the reconstruction of the entire system. And, any change either point charge or surrounding of charge affects the charge distribution on the entire surface. An event is defined as the occurrence of a certain sequence in the perception data. It is either predefined by the designer or a pattern learned by machine learning technology that initiates any driving mechanism. The agent in an agent-based system or a multi-agent-based system acts as an executor and determines actions based on perception data. The determinism of certain operations based on event definitions.

The complex process in the industrial automation unit will be subdivided (not hierarchical in nature), and eventually a set of atomic functions defined as distinct functions (DF) will be produced, and each DF is defined on one or more agents. The functional status of DF is as follows: start, process and terminate, and interrupt. The configuration is the current state of the associated entity and should be defined at multiple levels. The configuration level defines the hierarchical structure of the information flow, that is, the lowest level is from the surface level to the DF level from the DF level to the assembly level to the administrator level to the user level. Surface level is the surface defined for node $\mathrm{n}$, and its current configuration is determined by the state of node $\mathrm{n}$. Let us assume that the surface is defined as ten nodes, then the configuration of the surface will have the state of ten nodes. For the DF level, each DF has a surface state range defined by the designer as a configuration. The assembly level means that the assembly automation must have the instantaneous state of the entire DFs and surface. The administrative user should see the current state of the manufacturing process.

\subsection{Cyber-Physical System}

According to [16], more than thirty different technologies, as well as the participation of business and industry models, have achieved Industry 4.0. In essence, Industry 4.0 includes not only evolving business models, but also engineering and artificial intelligence. Some of the main players are discussed 
here as follows. Cyber physical system (CPS) provides a mechanism to monitor physical processes, the coordination of physical entities' perception, calculation, and communication. It is an engineering system monitored, controlled and integrated by the communication and computing core. CPS is a key participant in the realization of Industry 4.0 [17] and one of the main participants in providing other technologies for a wide range of applications such as Industry 4.0, industrial IoT and health networks. IoT technology is another key participant in the realization of Industry 4.0, which enables physical objects to communicate with other physical objects. This kind of object-to-object connectivity realizes the connectivity of systems, services, and physical objects, and realizes object-to-object communication and data sharing. It can realize the automation of different physical aspects, such as heating, lighting and remote control. Industry automation through the Internet-of-Services (IoS) in the digitalization of digital services or service providers can provide services. These services can be provided on demand around business models, partners, and any service settings. Suppliers provide services and aggregate them into value-added services because consumers can receive and access communications between consumers through various channels. Other participants are as follows: augmented reality, augmented reality, autonomous robots, additive manufacturing, cloud computing, and big data and analysis.

\subsection{Significance Data}

Data is the most important element of intelligent manufacturing; it can integrate the network world and the physical world [18]. Data is of fundamental significance for promoting Industry 4.0 from machine automation to information automation, and ultimately knowledge automation. For a zerofault-tolerant manufacturing industry, a fast control cycle is achieved through key data generation and communication. Faster sharing of data among production plants, operators, value chains, and other participants is a key requirement of Industry 4.0. Extracting information from data has a leading role in Industry 4.0, including operational data that is traditionally meaningless. In Industry 4.0, a large amount of data comes from different sources, namely NCS, IP-based cameras, physical measurement objects (CPS), etc. Some new concepts have been introduced, especially Industry 4.0, such as the concept of data bus and industrial data space for processing large amounts of factory data [19]. In order to understand the proposed model, a brief description of the concept of charge and its flux will be discussed in the next section.

\subsection{Physics of Point Charge Placed in a Closed Surface}

Electric flux can be defined as the number of current wires passing through a unit area. The electric flux from any surface caused by any charge can be determined from its charge distribution. A closed surface without any source charge has no electric field lines, that is, the electric field lines originate from the source charge and pass through it. Therefore, if there is no source charge in the enclosed surface, there will be no electric flux on the enclosed surface. In order to determine the electric flux that passes through the closed sphere of radius " $r$ " caused by the point charge $\mathrm{q}$, the dielectric constant $\varepsilon_{\mathrm{o}}$ shown in Fig. 2 defined by Gauss's law [20] is as follows,

$\varepsilon_{o}=1 /\left(4 \pi \varepsilon_{-} o r^{\wedge} 2\right)$

Electric flux passing through an area of sphere passing through " $\mathrm{A}$ " can be determined by [20],

$\varphi=E \cdot A \cos \theta$ 
The equation can be transformed into an equation which does not depends upon area and length as follows,

$\varphi=q / \varepsilon_{o}$

where $\varphi$ can be utilized to determine flux approaching towards the area in closed surface.

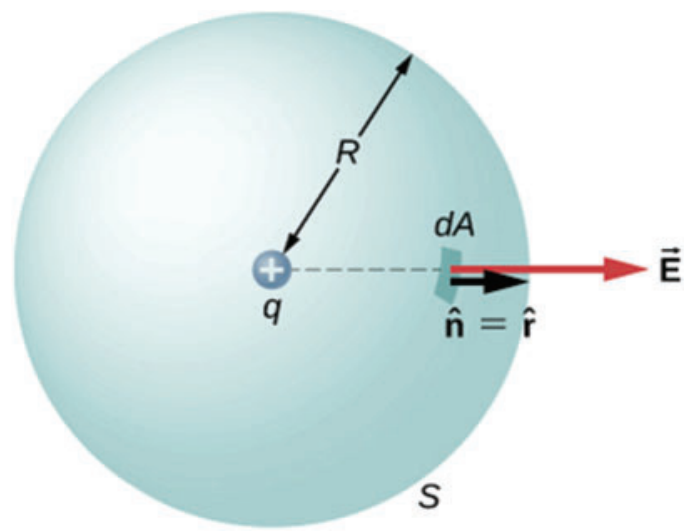

Figure 2: Electric flux through a closed sphere of a simple Gaussian surface, where $\mathrm{R}$ is radius of surface, $\vec{E}$ denotes electirc flux, where $\hat{n}=\hat{r}$ shows direction of electric flux, q denotes to point charge and $d A$ is magnitude of electric flux force [20]

\section{Proposed Analogy}

\subsection{Analogy and Structure Mapping Engine (SME)}

Qualitative reasoning is a way of expressing conceptual information, such as the structure of a physical system, causality, the beginning and end of a process, assumptions and facts that are true, behaviors of different nature, etc. This analogy is an example of qualitative reasoning. Analogy and similarity are important aspects of human cognition, affecting everything from visual perception to conceptual transformation. The comparative model must be able to interact with other processes and manage the scale and complexity of the representation required by the modeled activity to reflect its centrality.

Gentner's structural mapping theory is based on the concept that analogy is the mapping of knowledge from one domain (base) to another domain (target), which means that the relationship system established between basic projects is also established between target objects. Therefore, analogy is a way to discover the commonalities of relationships that have nothing to do with the objects that contain them. The concept of systematicity is at the core of the mapping process: individuals prefer to map a predicate system containing higher-order connections, rather than mapping isolated predicates. In interpreting analogies, systematic principles are structural expressions of our self-evident preferences for coherence and logical ability. In this framework, other types of similarity matches can be identified instead of analogies.

\subsection{Mapping of Domains}

Analogy is defined as the comparison or association between two objects or object systems, which highlights the similarities; analogy reasoning relies on analogy. Argument by analogy can be defined as reasoning that infers the existence of another similarity based on the similarity of the citation. The 
ability of human beings to solve problems is based on the ability of analogical reasoning. Analogical reasoning has always been a distinctive feature of all philosophical, scientific and legal reasoning. It is the best guide for all philosophical research, and all discoveries are made possible because of it [21].

An analogy is an application of one domain knowledge to another domain based on their relation similarity devised into a theory or framework for analogy [22,23]. The author, in their framework, defined analogy as "assentation that relation or structure normally applies in one domain can be applied to another domain." Two principles are defined in this analogy creation framework (a) mapping from source to target based on the relation between objects rather than object-attributes (b) Relation is defined systematically using higher-order logic. A simple analogy of the water reservoir and the electric battery can demonstrate abstract similarities required to build an analogy. In this example, a water reservoir is mapped on an electric battery, as shown in Tab. 1.

Table 1: Gentner's structure-mapping

\begin{tabular}{ll}
\hline Water reservoir & Electric battery \\
\hline Source for irrigation network & Source electric network \\
A reservoir is filled and spilled & The battery is charged and recharged \\
Pivotal position in the network & Pivotal position in the circuit \\
Well defined capacity & Well defined charging capacity \\
Water level & Charge level \\
\hline
\end{tabular}

Knowledge about the reservoir is applicable to battery; there exists a mapping based on similarity. The similarity in the reservoir battery example is literal similarity. However, Tversky designed a technique to determine the similarity between two domains, called the Tversky contrast model. Let A and $\mathrm{B}$ be two domains. The similarity between them should be higher than $\mathrm{A} \cap \mathrm{B}$ and smaller than their difference complement, namely $\mathrm{A}-\mathrm{B}$ and $\mathrm{B}-\mathrm{A}$. The structural mapping of knowledge from one domain to another is based on the three principles defined by Gentner's structural mapping.

Let $\mathrm{A}$ as analogy mapping from a base $\mathrm{B}$ to target $\mathrm{T}$ can be denoted as the equation shown below,

$A: B \rightarrow T$

where $\mathrm{B}$ is the domain, and each instance or object has a mapping with the target $\mathrm{T}$. The relationship between the retention and discarding of individual characteristics and the relationship between objects can be described in the form of the following equation,

$M: b_{i} \rightarrow t_{i}$

$M: b_{i} \nrightarrow t_{i}$

$M:\left(b_{i}, b_{j}, b_{k}\right) \rightarrow\left(b_{i}, b_{j}, b_{k}\right)$

Third and last principle postulate of framework is systematicity principle i.e.,

$R^{\prime}\left(R_{1}\left(b_{i}, b_{j}\right), R_{2}\left(b_{i}, b_{j}\right), \ldots, R_{n}\left(b_{i}, b_{j}\right)\right) \rightarrow, \ldots, R\left(R_{1}\left(b_{i}, b_{j}\right), R_{2}\left(b_{i}, b_{j}\right), \ldots, R_{n}\left(b_{i}, b_{j}\right)\right.$ 


\subsection{The Analogy of Point Charge with Sensing Node}

The point charge in the closed surface is responsible for the generation of electric flux on the closed surface, and the flux through the closed surface is only generated by the internal charge, that is, no external charge is responsible for the internal flux. In order to cope with the large complexity, the automation system is subdivided into multiple subsystems, which eventually become atomic functions. Each function has a sensing and actuation mechanism. The point charge is responsible for the electrical flux and at the same time senses the information flow of the node. Point charges continue to generate flux, that is, flux is generated in the form of flow, while sensor nodes continue to generate and transmit data. The point charge defines the boundary, and the sensor node has a clearly defined environment. Intensity changes change the flux evenly distributed over the entire surface (reflex action), while the latter perceives changes and broadcasts throughout the system (reflex action). The direction of the flow of positive charges remains on the outside, while the direction of the flow of negative charges is on the inside. For sensing, information flows to the outside, and for driving, it flows to the inside. Similarly, changes in charge intensity will eventually change the entire charge distribution (reconfiguration), and the quality of a good system determines the accuracy and efficiency of system recovery. The flux value is highly related to the material, that is, the dielectric constant of the material, and the information flow is highly dependent on the bandwidth of the communication medium. An analogy is given in Fig. 3. Although information architecture development should not be hierarchical; however, we designed a simplified symbolic model and its equivalent description to understand the topic, as shown in Fig. 4.

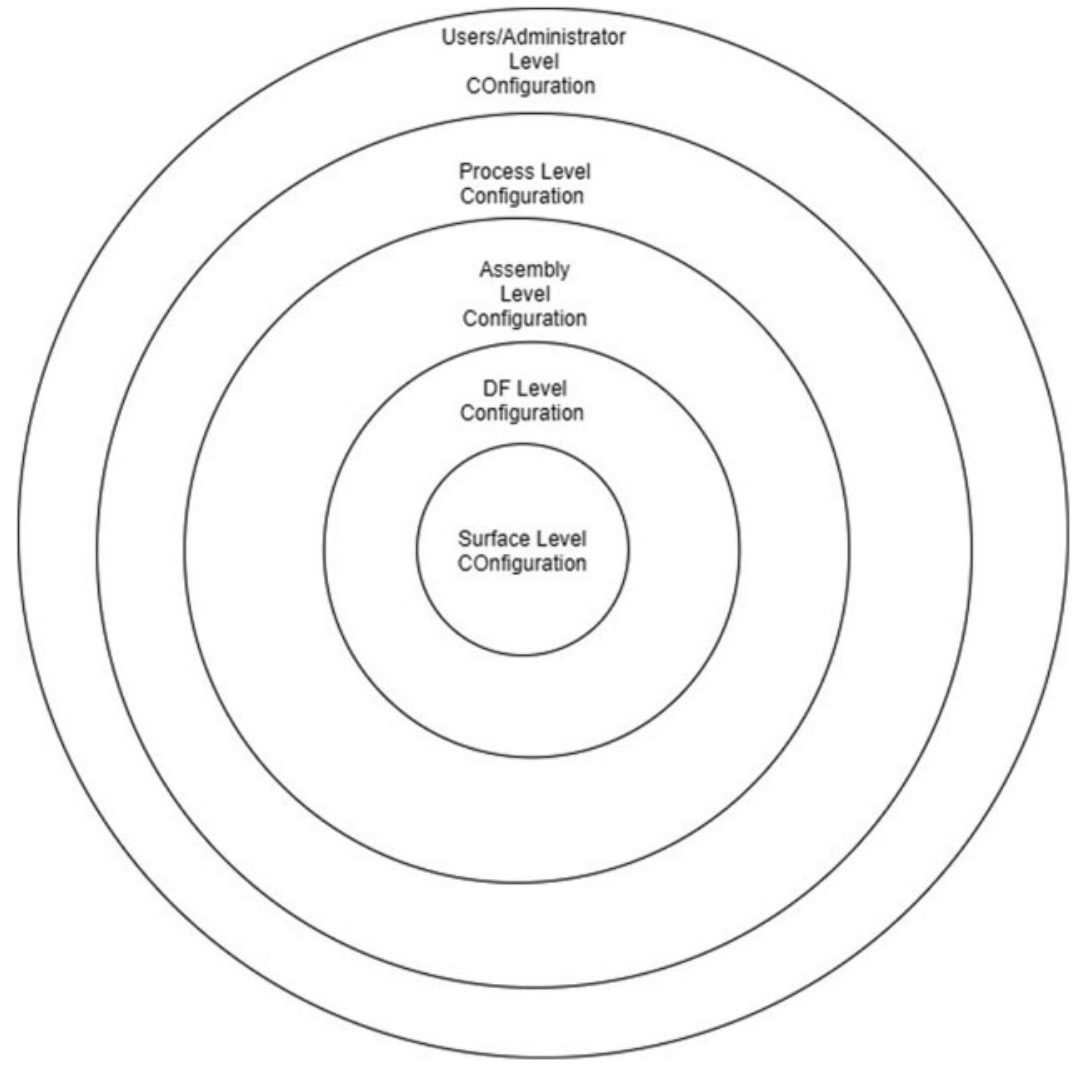

Figure 3: Hierarchal metaphor analogy 


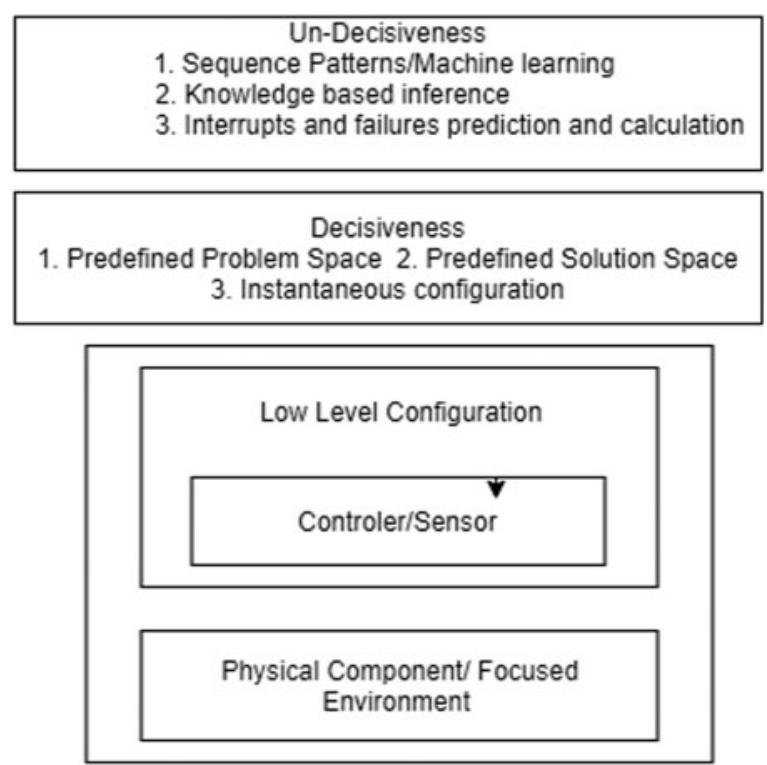

Figure 4: Scenario for analogy

\section{Structure-Mapping Pseudo-Codes}

In this section, we formally describe the analogy in pseudocode so that readers can understand it better. It includes different states of analogy and their counterparts.

Let us define both node and point charges as follows,

$N=(s, e, \omega, t)$

where $s$ is represents the set of states set, $e$ accounts for focused entity, $\omega$ is the range of possible sensing values, and $t$ is the time. However, the point charge is defined as follows,

$Q=(q, s, i, t)$

where $q$ is charge type and $s$ is denotes surface of the enclosed body, $i$ is an intensity of the charge at any time $t$. A change in the state can be defined as follows,

$C_{s}=[0, \omega]$

where $\mathrm{C}_{\mathrm{s}}$ is used to sense the change of node state, and $\omega$ is the highest possible value that can be sensed. The term "event" in the real world is used as a general term to describe free and loose things and their occurrence. For the scientific term event, it is related to time, that is, the event lasts for a period of time (no matter how long or how short). For example, the four-year war and the emission and transmission of photons from the light source within picoseconds are clearly defined events [24]. On the other hand, in information and artificial intelligence, the term "event" is defined as "a change or sequence of changes in a centralized environment, which has defined and undefined consequences."

For the traditional event-driven architecture used in information science, events are atomic and plot in nature (events either happen or not happen, but not in a partial sense). Events have the properties of initiate, hold, and actuate. The definition of an event is given below,

$E_{1}=\left\{C_{1}, C_{2}, C_{3}, \ldots, C_{n}\right\}$ 
where $\mathrm{E} 1$ defined as sequence of change $\because E_{1} \neq \emptyset$. Further agent function is defined as follows,

$f(c, a)=\left\{\left(c_{1}, a_{1}\right),\left(c_{2}, a_{2}\right),\left(c_{3}, a_{3}\right), \ldots,\left(c_{n}, c_{n}\right)\right\}$

where $c$ is a sequence of change and $a$ action (reflex actions) defined by the developer. The complete agent function procedure is described in Algorithm 1.

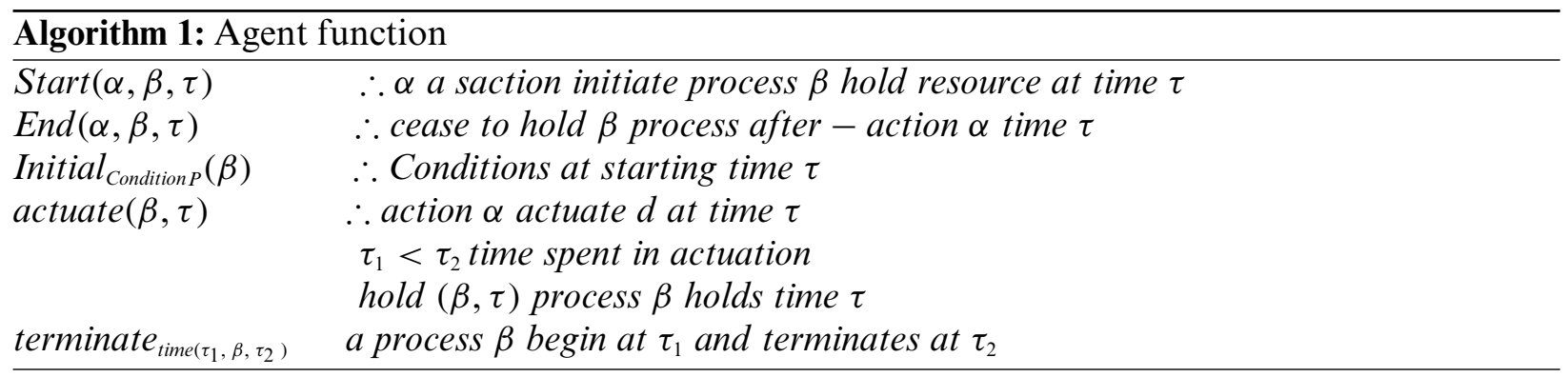

And theoretical actuation sequence is given in Eq. (21) up to Eq. (23)

actuate $(, \tau) \leftarrow \operatorname{Initial}_{\text {condition }}(c) \wedge \neg$ terminate $\left(\tau_{0}, c, \tau_{1}\right)$

hold $\left(c, \tau_{2}\right)$ hold next time

...actuate $\left(a, \tau_{1}\right) \wedge$ starts $\left(a, c, \tau_{1}\right) \wedge \tau_{1}<\tau_{2} \wedge \neg$ terminate $\left(\tau_{1}, c, \tau_{2}\right)$

terminate $\left(\tau_{1}, c, \tau_{2}\right)$

$\ldots \exists a, t\left[\right.$ actuate $(c, \tau) \wedge \tau_{1}<\tau<\tau_{2} \wedge$ terminate $\left(\tau_{1}, c, \tau_{2}\right)$

According to the second hypothesis of the analogy (defined as the surface area of the event should be regarded as the point charge in the closed sphere), this change in the surface area A should be communicated according to the new next layer, and the new sequence of changes and this event can be defined as one Group event identification and status, holding resources and time start and end are as follows,

$C_{E}=\left\{\left(\right.\right.$ Event_State, $\beta_{i}$, terminate $)$ te - time $\}$

while event state can be defined as $\operatorname{Start}(\alpha, \beta, \tau), \operatorname{End}(\alpha, \beta, \tau), \operatorname{Initial}_{\text {Condition } P}(\beta), \operatorname{actuate}(\alpha, \tau), \tau_{1}<$ $\tau_{2}, \operatorname{hold}(\beta, \tau)$. These change values modify the state variables of the upper layer, thereby affecting the change events scattered in the entire focusing environment. This not only affects the state value of each surface, but also modifies the conditions of the agent area $\mathrm{A}$ on each enclosed surface as follows,

$M: A \rightarrow T$ 
The source domain point charge is contained in the closed surface asset $\mathrm{A}$, while the target domain sensing node is placed in the predefined environmental asset $\mathrm{T}$. The first step of the mapping framework is to discard the attributpes of the source domain and the target domain, which are defined as follows,

$A(q, \varepsilon, s, i, f l)]-\rightarrow A^{\prime}[(n, \theta, a, s, c h)$

where $q$ stands for a charge, $\varepsilon$ is the permittivity of the medium, $\mathrm{s}$ is surface, $i$ is the intensity of medium $f l$ accounts for electric flow lines. Moreover, $\mathrm{n}$ is the sensing node and $\theta$ is bandwidth limitations, $a$ is the area of focused environment, $s$ is set of states predefined, and $c h$ is defined as channel medium. We now define relationships in the following,

$R(q, i),(\varepsilon, i),(s, f l)] \rightarrow R^{\prime}[(n, s),(\theta, s)$

Final systematic mapping of attributes from source to target is shown as follows,

$R\{R(q, i), R(\varepsilon, i), \ldots,\} \rightarrow R^{\prime}\left\{R^{\prime}(n, s),(\theta, s), \ldots,\right\}$

\section{Discussions}

Reconfiguration is the basic requirement of CPS-based industrial automation environment. The smart grid environment enables the system to communicate reconfigurations in the event of unpredictable sudden and random changes. These changes need to be communicated to the entire system at a higher speed because electrical phenomena appear faster in the system. Therefore, the entire system needs to be configured and reconfigured on the fly. For an uncertain environment where the product line should be highly personalized, with minimal cost and time constraints, solutions for information generation, flow, and result calculation should meet hard real-time standards. The realization of cyber-physical systems for industrial automation utilizes an event-driven architecture based on multi-agents. The change and generation of an event need a spontaneous mechanism to propagate through the network and calculations. This research provides a solution similar to the propagation of electric flux through a closed surface. With the charge distribution as the configuration of the system, any change in charge will immediately change the charge distribution on the entire surface.

This analogy provides a theoretical basis for the development of information architecture based on the physical phenomenon of the electric flux passing through a hollow sphere (Gaussian sphere) due to a point charge. Because the information architecture of the smart grid system needs to meet multiple requirements of information, so that the system can detect, react and actively respond to changes in the system at the same speed when power occurs. This analogy will provide the basis for the development of information architecture with the ability to report electrical phenomena at the rate of occurrence.

\section{Conclusions}

This research paper provides an analogy of current passing through a charged hollow sphere due to a point charge. Each node is similar to a point charge, and its surface is similar to the next layer of nodes. Electric flux is similar to the communication channel between nodes. This analogy is based on the fact that every Gaussian sphere retains the same charge. For simplicity, we use an example of a smart grid, where the information requirements are difficult to implement in real time. The smart grid system is a conceptual power system that can realize bidirectional current. Its digital 
communication supports real-time detection, reaction and proactive action on any changes in the entire system, and has self-repair capabilities. It also supports instant communication with suppliers and consumers. The smart grid system needs an IA that supports communication requirements to report the hard real-time changes that follow the power in the system. In addition, smart grid systems need to report changes on either side of the system (i.e., consumers and suppliers) in order to configure and reconfigure the system based on changes that occur. This research provides a solution for a spontaneously changing environment, which defines changes and their sequence (events) and the mechanism of configuration and reconfiguration; as for the concerns, it is recommended that the work divide them into two categories, (pre-determined and undecided). Our analogy provides an interesting opportunity for the development of a modern I.4-based system that can realize instant configuration and spontaneous information exchange for real-world data requirements, especially the realization of hard real-time implementation. We use the well-known Gentner framework to prove the analogy and the structure mapping engine framework to support the evidence of proof. The mapping of the point charges enclosed in the enclosed surface as the source and sensing nodes/process components in the environment has a maximum value, but is within the boundaries of Gentner's analogy mapping framework. The analogy we propose provides a more general idea for building the information architecture of such systems in a dynamic environment.

Acknowledgement: We deeply acknowledge Taif University for supporting this study through Taif University Researchers Supporting Project Number (TURSP-2020/150), Taif University, Taif, Saudi Arabia.

Funding Statement: This work was supported by Taif University (in Taif, Saudi Arabia) through the Researchers Supporting Project Number (TURSP-2020/150).

Conflicts of Interest: The authors declare that they have no conflicts of interest to report regarding the present study.

\section{References}

[1] T. P. Raptis, A. Passarella and M. Conti, "Data management in industry 4.0: State of the art and open challenges," IEEE Access, vol. 7, pp. 97052-97093, 2019.

[2] M. Rüßmann, M. Lorenz, P. Gerbert, M. Waldner, J. Justus et al., "Industry 4.0: The future of productivity and growth in manufacturing industries," Boston Consulting Group, vol. 9, no. 1, pp. 54-89, 2015.

[3] A. Theorin, K. Bengtsson, J. Provost, M. Lieder, C. Johnsson et al., "An event-driven manufacturing information system architecture for industry 4.0," International Journal of Production Research, vol. 55, no. 5, pp. 1297-1311, 2017.

[4] Y. Lu, "Industry 4.0: A survey on technologies, applications, and open research issues," Journal of Industrial Information Integration, vol. 6, pp. 1-10, 2017.

[5] J. Wan, S. Tang, Z. Shu, D. Li, S. Wang et al., "Software-defined industrial internet of things in the context of industry 4.0," IEEE Sensors Journal, vol. 16, no. 20, pp. 7373-7380, 2016.

[6] N. Jazdi, "Cyber physical systems in the context of industry 4.0," in Proc. IEEE Int. Conf. on Automation, Quality and Testing, Robotics, Cluj-Napoca, Romania, pp. 1-4, 2014.

[7] Z. Ge, Z. Song, S. X. Ding and B. Huang, "Data mining and analytics in the process industry: The role of machine learning," IEEE ACCESS, vol. 5, pp. 20590-20616, 2017.

[8] A. Theorin, K. Bengtsson, J. Provost, M. Lieder, C. Johnsson et al., "An event-driven manufacturing information system architecture for industry 4.0," International Journal of Production Research, vol. 55, no. 5, pp. 1297-1311, 2017. 
[9] K. Gai, Z. Lu, M. Qiu and L. Zhu, "Toward smart treatment management for personalized healthcare," IEEE Access, vol. 33, no. 6, pp. 30-36, 2019.

[10] K. Gai and M. Qiu, "Reinforcement learning-based content-centric services in mobile sensing," IEEE Network, vol. 32, no. 4, pp. 34-39, 2018.

[11] F. García-Muiña, E. Medina-Salgado, M. S. Ferrari and M. Cucchi, "Sustainability transition in industry 4.0 and smart manufacturing with the triple-layered business model canvas," Sustainability, vol. 12, no. 6, pp. 2364, 2020.

[12] J. Butt, "Exploring the interrelationship between additive manufacturing and industry 4.0," Designs, vol. 4, no. 2, pp. 13, 2020.

[13] M. Merdan, T. Hoebert, E. List and W. Lepuschitz, "Knowledge-based cyber-physical systems for assembly automation," Production and Manufacturing Research, vol. 7, no. 1, pp. 223-254, 2019.

[14] D. Regulin, D. Schütz, T. Aicher and B. Vogel-Heuser, "Model-based design of knowledge bases in multiagent systems for enabling automatic reconfiguration capabilities of material flow modules," in Proc. Int. Conf. on Automation Science and Engineering, Texas, USA, pp. 133-140, 2016.

[15] S. Eisele, I. Mardari, A. Dubey and G. Karsai, "RIAPS: Resilient information architecture platform for decentralized smart systems," in Proc. IEEE Int. Symp. on Real-Time Distributed Computing, Toronto, Canada, pp. 125-132, 2017.

[16] D. S. Batra and D. Juneja, "M-commerce in India: Emerging issues," International Journal of Advanced Research in IT and Engineering, vol. 2, no. 2, pp. 54-65, 2013.

[17] J. Lee, H. A. Kao and S. Yang, "Service innovation and smart analytics for industry 4.0 and big data environment," in Proc. Conf. on Industrial Product-Service Systems, Ontario, Canada, vol. 16, pp. 3-8, 2014.

[18] B. Otto and M. Jarke, "Designing a multi-sided data platform: Findings from the international data spaces case," Electronic Markets, vol. 29, no. 4, pp. 561-580, 2019.

[19] D. Wang, "Building value in a world of technological change: Data analytics and industry 4.0," IEEE Engineering Management Review, vol. 46, no. 1, pp. 32-33, 2018.

[20] S. J. Ling and J. Sanny, "University physics II-thermodynamics, electricity, and magnetism (OpenStax), Texas, USA: Rice University Houston,”2020. [Online]. Available: https://phys.libretexts.org/@go/pa ge/4343.

[21] A. Chakrabortty and A. Bose, "Smart grid simulations and their supporting implementation methods," Proceedings of IEEE, vol. 105, no. 11, pp. 2220-2243, 2017.

[22] R. J. Sternberg and B. Rifkin, "The development of analogical reasoning processes," Journal of Experimental Child Psychology, vol. 27, no. 2, pp. 195-232, 1979.

[23] D. Gentner, "Structure-mapping: A theoretical framework for analogy," Cognitive Science, vol. 7, no. 2, pp. $155-170,1983$.

[24] P. M. All and J. Terms, "Determinism, indeterminism and the flow of time," Erkenntnis, vol. 56, no. 2, pp. 123-150, 2002. 